\title{
Zinc finger E-box binding homeobox 2 functions as an oncogene in human laryngeal squamous cell carcinoma
}

\author{
QUN LI ${ }^{1}$, LIANG MA ${ }^{2}$, ZHENHUA WU ${ }^{3}$, GUOLI WANG ${ }^{1}$, QI HUANG ${ }^{3}$, ZHISEN SHEN $^{1}$ and RUI YU ${ }^{2}$ \\ ${ }^{1}$ Department of Otorhinolaryngology, Head and Neck Surgery, Lihuili Hospital of Ningbo University, Ningbo, \\ Zhejiang 315040; ${ }^{2}$ Department of Biochemistry, School of Medicine, Ningbo University, Ningbo, Zhejiang 315211; \\ ${ }^{3}$ Department of Otorhinolaryngology Head and Neck Surgery, Ningbo Medical Center, \\ Lihuili Eastern Hospital, Ningbo, Zhejiang 315030, P.R. China
}

Received September 24, 2017; Accepted December 4, 2018

DOI: $10.3892 / \mathrm{mmr} .2019 .10126$

\begin{abstract}
Zinc finger E-box binding homeobox 2 (ZEB2) is a member of the Zfh1 family of two-handed zinc finger/homeodomain proteins. To date, the role of ZEB2 in human laryngeal carcinoma has not been clearly defined. In the present study, the level of ZEB2 expression in laryngeal squamous cell carcinoma (LSCC) tissues and adjacent normal tissues was evaluated using reverse transcription-quantitative polymerase chain reaction. The effects of ZEB2 on the growth, migration, invasion, cell cycle distribution and apoptosis of laryngeal cancer cells were also explored using MTT, Transwell and flow cytometry assays. It was identified that ZEB2 was upregulated in LSCC tissues compared with normal tissues. Silencing of ZEB2 inhibited the viability, migration and invasion of LSCC cells. It was also observed that ZEB2 silencing induced cell cycle arrest and apoptosis in LSCC cells. Furthermore, ZEB2 silencing inhibited the process of epithelial-mesenchymal transition. Overall, the results indicated that ZEB2 promotes the progression of LSCC and that it may be a potential target for the treatment of this type of cancer.
\end{abstract}

\section{Introduction}

Laryngeal cancer is one of the most commonly observed malignancies of the head and neck, and accounts for $25 \%$ of

Correspondence to: Dr Zhisen Shen, Department of Otorhinolaryngology, Head and Neck Surgery, Lihuili Hospital of Ningbo University, 57 Xingning Road, Ningbo, Zhejiang 315040, P.R. China

E-mail: szs7216@163.com

Dr Rui Yu, Department of Biochemistry, School of Medicine, Ningbo University, 818 Fenghua Road, Ningbo, Zhejiang 315211, P.R. China

E-mail: m18721550175@163.com

Key words: zinc finger E-box binding homeobox 2, laryngeal squamous cell carcinoma, cell cycle, apoptosis, epithelial-mesenchymal transition tumors in this region (1). Laryngeal cancer accounts for $\sim 1.1 \%$ of all newly diagnosed cancer cases and for nearly $1 \%$ of all tumor-associated mortalities worldwide (2). Among different subtypes, laryngeal squamous cell carcinoma (LSCC) accounts for $>90 \%$ of laryngeal cancer cases, and is more prevalent in middle-aged and elderly males (3). Although advances have been achieved in the management of this tumor, the survival rates of patients with LSCC have remained unfavorable for the past 30 years (4). Therefore, it is urgent to unveil the molecular mechanisms underlying the development of LSCC.

Zinc finger E-box binding homeobox 2 (ZEB2), a transcription factor that belongs to the Zfh1 family, has been found to be overexpressed in various types of cancer, including colorectal, prostate and pancreatic cancer (5-7). Mounting evidence has indicated that ZEB2 is involved in the initiation and development of malignancies (8). Furthermore, it has been reported that ZEB2 promotes the epithelial-mesenchymal transition (EMT), a pathological process leading to specific morphological and phenotypic alterations during cancer metastasis in tumor cells $(9,10)$. According to previous studies, ZEB2 was significantly upregulated in $\operatorname{LSCC}(11,12)$. However, the function of ZEB2 in LSCC remains largely unknown.

In the present study, the expression of ZEB2 in LSCC and adjacent normal tissues was investigated. In addition, the effect of ZEB2 silencing on the proliferation, migration, invasion, cell cycle distribution, apoptosis and EMT of LSCC cells was examined. The results revealed that ZEB2 functioned as an oncogene in LSCC and that targeting ZEB2 may be a promising therapeutic target in the management of LSCC.

\section{Patients and methods}

Patients and specimens. Between January 2014 and February 2015, laryngeal cancer tissues $(n=46)$ and the corresponding adjacent non-tumor tissues $(n=46)$ were collected from laryngeal cancer patients in the Department of Otorhinolaryngology, Head and Neck Surgery (Lihuili Hospital, Ningbo, China). Patient characteristics are presented in Table I. There were 21 female and 25 male patients, aged from 40-79 and the mean age was 59. All patients were diagnosed with LSCC according to WHO criteria (13). The research was approved by the Institutional Review Board of Lihuili Hospital, 
and written informed consent was obtained from each patient prior to surgery.

Cell culture. Human laryngeal cancer AMC-HN8 cells (cat no. 0143321) were obtained from the Shanghai Cell Bank of the Chinese Academy of Science (Shanghai, China). Cells were cultured in RPMI-1640 medium (Gibco; Thermo Fisher Scientific, Inc., Waltham, MA, USA) with $10 \%$ fetal bovine serum (FBS; Gibco; Thermo Fisher Scientific, Inc.) at $37^{\circ} \mathrm{C}$ with $5 \% \mathrm{CO}_{2}$.

Reverse transcription-quantitative polymerase chain reaction $(R T-q P C R)$. Total RNA was extracted from the cells or tissues by TRIzol reagent (Thermo Fisher Scientific, Inc.). The quality and concentration of RNA was measured by NanoDrop (Thermo Fisher Scientific, Inc.). RNA samples were reverse transcribed using cDNA synthesis kit (Invitrogen; Thermo Fisher Scientific, Inc.) according to the manufacturer's protocols. qPCR was then performed using the SYBR ExScript qRT-PCR kit (Takara Biotechnology Co., Ltd., Dalian, China) and conducted on an Applied Biosystems 7500 Real-Time PCR system (Thermo Fisher Scientific, Inc.). Conditions of the PCR amplification were: $94^{\circ} \mathrm{C}(5 \mathrm{~min})$, then 30 cycles at $94^{\circ} \mathrm{C}$ $(30 \mathrm{sec}), 60^{\circ} \mathrm{C}(45 \mathrm{sec})$ and $72 \mathrm{C}(45 \mathrm{sec})$ with a final extension at $72 \mathrm{C}$ for $10 \mathrm{~min}$.

The primer pairs used in qPCR were as follows: ZEB2 forward, 5'-AAGGAGCAGGTAATCGCAAG-3', and reverse, 5'-GGA ACCAGAATGGGAGAAACG-3'; GAPDH forward, 5'-AGA AGGCTGGGGCTCATT TG-3' and reverse, 5'-AGGGGCCATCCACAGTCTTC-3'. Glyceraldehyde-3-phosphate dehydrogenase (GAPDH) was used as the endogenous control. The expression fold changes were calculated using the $2^{-\Delta \Delta \mathrm{Cq}}$ method (14).

Silencing of ZEB2. ZEB2 was silenced in AMC-HN8 cells with two different small interfering RNAs (siRNAs), which were synthesized by GeneChem Co., Ltd. (Shanghai, China). The siRNA sequences were as follows: si-ZEB2-1, 5'-TGA AAACATAGTCCCCAACA-3'; and si-ZEB2-2, 5'-CTG GACAACAAAAGCACT-3'. Briefly, cells were seeded at the density of $1 \times 10^{6}$ cells/well in 6-well plates and incubated until $70 \%$ confluency was reached. On the following day, the appropriate amount of siRNA against ZEB2 (si-ZEB2-1 and si-ZEB2-2) or negative control siRNA (si-NC) were transfected into the cells using Lipofectamine 3000 reagent (Thermo Fisher Scientific, Inc.) according to the manufacturer's protocol.

Cell proliferation assay. Cell viability was evaluated by an MTT assay. Briefly, cells were seeded in a 96 -well plate $\left(5 \times 10^{3}\right.$ cells/well) and then treated for $24 \mathrm{~h}$ with or without siRNAs. A total of $50 \mu \mathrm{l}$ MTT solution $(5 \mathrm{mg} / \mathrm{ml})$ was added to each well, and the cells were incubated for $4 \mathrm{~h}$. The medium was then removed, and $200 \mu \mathrm{l}$ dimethyl sulfoxide (DMSO) was added to each well. Subsequently, the absorbance of the solutions was measured on a BioTek microplate reader (BioTek Instruments, Inc., Winooski, VT, USA) at $595 \mathrm{~nm}$. The relative cell viability (\%) was measured by comparing the absorbance at $595 \mathrm{~nm}$ with that of the vehicle control, which was treated with $0.1 \%$ DMSO.
Cell cycle distribution analysis. Cells grown in 6-well plates $\left(1 \times 10^{6}\right.$ cells/well) were treated with siRNA against ZEB2 or si-NC. The floating cells were discarded, and attached cells were harvested and fixed in ice-cold $70 \%$ ethanol. Next, the fixed cells were washed with phosphate-buffered saline (PBS) and resuspended in PBS containing RNase A $(20 \mu \mathrm{g} / \mathrm{ml})$. The cells were fixed with $70 \%$ ethanol and stained with propidium iodide (PI), and the cell cycle distribution was evaluated using FACSVerse ${ }^{\mathrm{TM}}$ flow cytometer (Beckman Coulter, Inc., Fullerton, CA, USA). Data were analyzed using FlowJo version 10 software (FlowJo LLC, OH, USA).

Apoptosis assay. Cells grown in 6-well plates were treated with siRNA against ZEB2 or si-NC. All the cells were harvested and fixed in ice-cold $70 \%$ ethanol. The fixed cells were then washed with PBS, resuspended in PBS containing RNase A $(20 \mu \mathrm{g} / \mathrm{ml})$ and stained with the Annexin V-FITC/PI Apoptosis Detection kit (BD Biosciences, Franklin Lakes, NJ, USA). Cell apoptosis was evaluated using FACSVerse ${ }^{\mathrm{TM}}$ flow cytometer, and data were analyzed using FlowJo version 10 software.

Cell migration and invasion assays. For the migration assays, at $48 \mathrm{~h}$ post-transfection, $5 \times 10^{4}$ cells in serum-free medium were placed into the upper chamber of a Transwell insert (24-wells plate, $8-\mu \mathrm{m}$ pore size; EMD Millipore, Billerica, MA, USA). For the invasion assays, at $48 \mathrm{~h}$ post-transfection, $1 \times 10^{5}$ cells in serum-free medium were placed into the upper chamber of a Transwell insert coated with Matrigel (Sigma-Aldrich; Merck KGaA, Darmstadt, Germany). Medium containing $10 \%$ FBS was added to the lower chamber for both assays. Subsequent to incubation for $24 \mathrm{~h}$, the cells remaining on the upper membrane were removed with cotton wool. Cells that had migrated or invaded through the membrane were stained with methanol and $0.1 \%$ crystal violet, and counted under an inverted microscope (Olympus Corporation, Tokyo, Japan). Experiments were independently repeated three times.

Western blot assay. Following transfection, cells were lysed with radioimmunoprecipitation assay buffer (Beyotime Institute of Biotechnology, Shanghai, China). The concentration of protein lysates were assayed by the Bradford kit (Beyotime Institute of Biotechnology, Shanghai, China). The lysates (40 $\mu \mathrm{g})$ were resolved by $12 \%$ SDS-PAGE and transferred to a polyvinylidene difluoride membrane. The membrane was blocked with $10 \%$ skimmed-milk at room temperature for $1 \mathrm{~h}$. Subsequently, the membranes were incubated overnight at $4^{\circ} \mathrm{C}$ with primary antibodies against the following: ZEB2 (23312), obtained from Wuhan Sanying Biotechnology (Wuhan, China); matrix metalloproteinase MMP-2 (ab37150), MMP-9 (ab58803), E2 promoter binding factor 1 (E2F1) (ab179445) and vimentin (ab137321), purchased from Abcam (Cambridge, MA, USA); p21 (2947), cyclin D1 (2922), cyclin-dependent kinase (CDK)4 (12790), CDK6 (3136), caspase-3 (9662), caspase-8 (9746), caspase-9 (9502), cleaved poly(ADP-ribose) polymerase (PARP) (9541), B-cell lymphoma 2 (Bcl-2) (4223) and Bcl-2-associated X protein (Bax) (2774), all purchased from Cell Signaling Technology, Inc. (Danvers, MA, USA); E-cadherin (743712) and N-cadherin (746629), from BD Biosciences; and GAPDH (G5262), obtained from Sigma-Aldrich (Merck KGaA). The samples were then incubated with the appropriate secondary 
Table I. Clinical characteristics of 46 laryngeal squamous cell carcinoma patients.

\begin{tabular}{|c|c|c|c|}
\hline Specimen no. & $\begin{array}{c}\text { Histologic } \\
\text { differentiation }\end{array}$ & $\begin{array}{c}\text { Age } \\
\text { (years) }\end{array}$ & TNM stage \\
\hline 1 & Poor & 76 & T1N0M0 \\
\hline 2 & Moderate & 53 & T4N0M0 \\
\hline 3 & Moderate & 64 & T1N0M0 \\
\hline 4 & Moderate & 49 & T1N0M0 \\
\hline 5 & Poor & 64 & T4N2M0 \\
\hline 6 & Good & 57 & T3N0M0 \\
\hline 7 & Poor & 57 & T4N0M0 \\
\hline 8 & Moderate & 64 & T1N0M0 \\
\hline 9 & Moderate & 57 & T4N2M0 \\
\hline 10 & Poor & 61 & T4N1M0 \\
\hline 11 & Poor & 56 & $\mathrm{~T} 3 \mathrm{~N} 2 \mathrm{M} 0$ \\
\hline 12 & Good & 70 & T3N0M0 \\
\hline 13 & Good & 51 & T4N1M0 \\
\hline 14 & Moderate & 59 & T4N1M0 \\
\hline 15 & Poor & 68 & $\mathrm{~T} 2 \mathrm{~N} 1 \mathrm{M} 0$ \\
\hline 16 & Poor & 79 & T1N0M0 \\
\hline 17 & Poor & 55 & T4N1M0 \\
\hline 18 & Good & 48 & T4N2M0 \\
\hline 19 & Moderate & 59 & T3N2bM0 \\
\hline 20 & Poor & 67 & T1N0M0 \\
\hline 21 & Poor & 59 & $\mathrm{~T} 2 \mathrm{~N} 1 \mathrm{M} 0$ \\
\hline 22 & Moderate & 64 & T1N0M0 \\
\hline 23 & Moderate & 57 & T1N0M0 \\
\hline 24 & Good & 52 & T1N0M0 \\
\hline 25 & Poor & 48 & T1N0M0 \\
\hline 26 & Poor & 60 & T1N0M0 \\
\hline 27 & Moderate & 54 & T1N0M0 \\
\hline 28 & Good & 40 & T2N0M0 \\
\hline 29 & Good & 45 & T2N0M0 \\
\hline 30 & Moderate & 63 & T3N2bM0 \\
\hline 31 & Poor & 51 & T1N0M0 \\
\hline 32 & Poor & 62 & $\mathrm{~T} 2 \mathrm{~N} 2 \mathrm{MO}$ \\
\hline 33 & Good & 51 & T3N1M0 \\
\hline 34 & Moderate & 59 & T4N1M0 \\
\hline 35 & Good & 63 & $\mathrm{~T} 2 \mathrm{~N} 2 \mathrm{M} 0$ \\
\hline 36 & Moderate & 74 & $\mathrm{~T} 3 \mathrm{~N} 1 \mathrm{M} 0$ \\
\hline 37 & Poor & 52 & T1N0M0 \\
\hline 38 & Poor & 72 & $\mathrm{~T} 4 \mathrm{~N} 2 \mathrm{M} 0$ \\
\hline 39 & Moderate & 63 & T2N0M0 \\
\hline 40 & Moderate & 60 & T1N0M0 \\
\hline 41 & Good & 51 & T4N0M0 \\
\hline 42 & Good & 51 & T3N1M0 \\
\hline 43 & Moderate & 55 & T1N0M0 \\
\hline 44 & Moderate & 54 & T1N0M0 \\
\hline 45 & Poor & 66 & T1N0M0 \\
\hline 46 & Poor & 54 & T1N0M0 \\
\hline
\end{tabular}

antibodies at room temperature for $1 \mathrm{~h}$ (Sigma-Aldrich; Merck KGaA). Signals were visualized using an enhanced

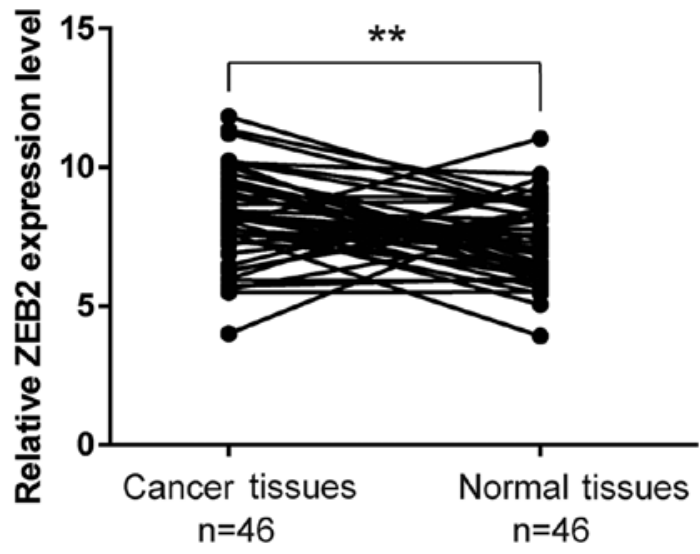

Figure 1. ZEB2 was significantly upregulated in laryngeal cancer tissues. ZEB2 mRNA levels were examined in laryngeal cancer tissues $(n=46)$ and the corresponding non-tumor tissues by reverse transcription-quantitative polymerase chain reaction, and the results were normalized to GAPDH expression. P-values between samples were calculated by performing a t-test. ${ }^{* *} \mathrm{P}<0.01$. ZEB2, zinc finger E-box binding homeobox 2 .

chemiluminescence reagent (Pierce; Thermo Fisher Scientific, Inc.).

Statistical analyses. All the experiments were conducted at least three times. The data were analyzed using GraphPad Prism version 6.0 software (GraphPad Software, Inc., La Jolla, CA, USA). The results are presented as the mean \pm standard deviation, and the differences between two groups or multiple groups were measured using Student's t-test or using analysis of variance followed by Tukey's test, respectively. $\mathrm{P}<0.05$ was considered to indicate a statistically significant difference.

\section{Results}

ZEB2 is significantly upregulated in laryngeal cancer tissues. ZEB2 expression levels were investigated using RT-qPCR assays in 46 laryngeal cancer tissues and adjacent histologically normal tissues. The results demonstrated that ZEB2 expression was significantly higher in tumor tissues compared with that in adjacent normal tissues $(\mathrm{P}<0.01$; Fig. 1).

Silencing ofZEB2 represses laryngeal cancercellproliferation, migration and invasion in vitro. To investigate the potential role of ZEB2 in laryngeal cancer cell proliferation, two siRNAs against ZEB2 were transfected into laryngeal cancer AMC-HN8 cells. The RT-qPCR and western blot analyses confirmed that the expression of ZEB2 was effectively reduced following transfection (Fig. 2A). The MTT assay demonstrated that the proliferation of AMC-HN8 cells was significantly suppressed by ZEB2 downregulation, as compared with that in the si-NC group (Fig. 2B). The role of ZEB2 in cell migration and invasion was then further evaluated. As shown in Fig. $2 \mathrm{C}$ and D, ZEB2 silencing resulted in marked repression of the migration and invasion abilities of AMC-HN8 cells $(\mathrm{P}<0.01)$. It was also observed that the protein expression levels of MMP-2 and MMP-9 were downregulated following silencing of ZEB2 (Fig. 2E). 
A

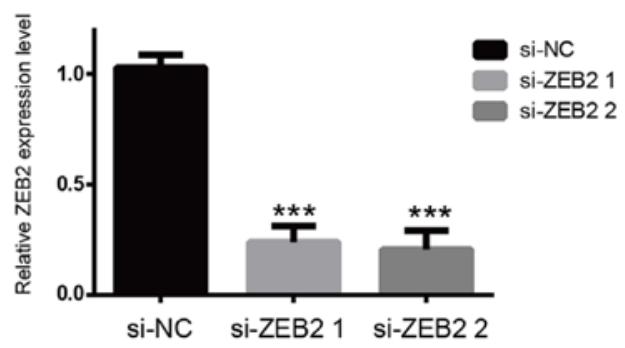

ZEB2

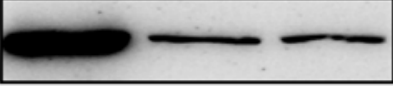

GAPDH

C
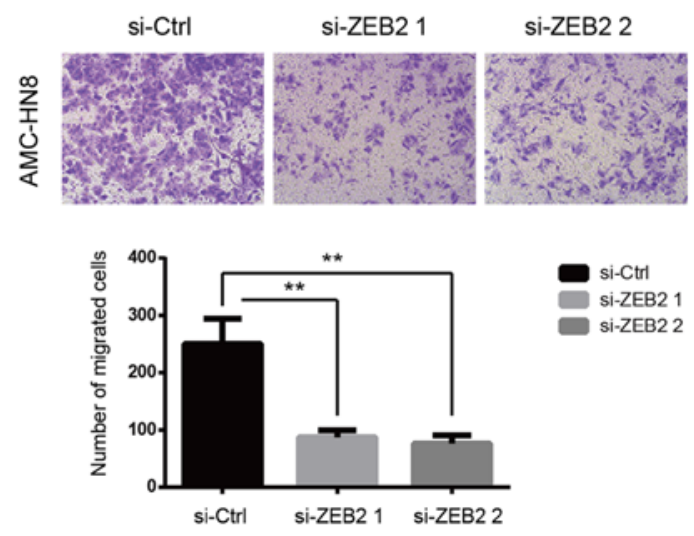

$E$

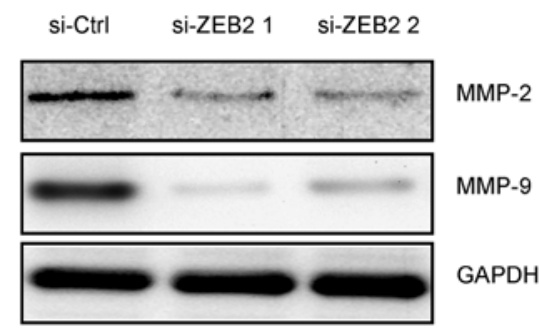

B

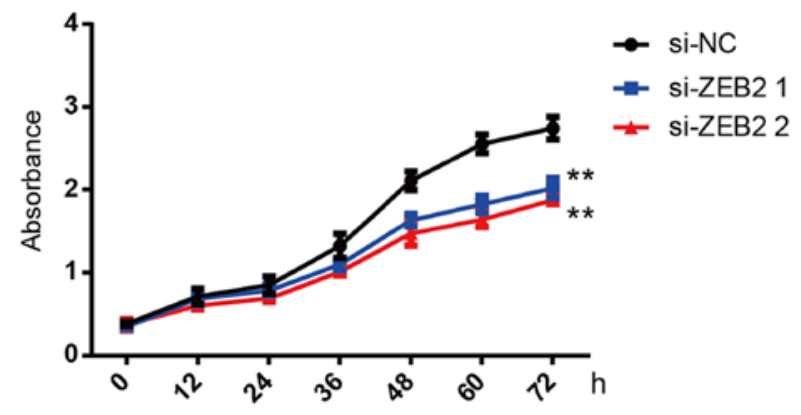

D

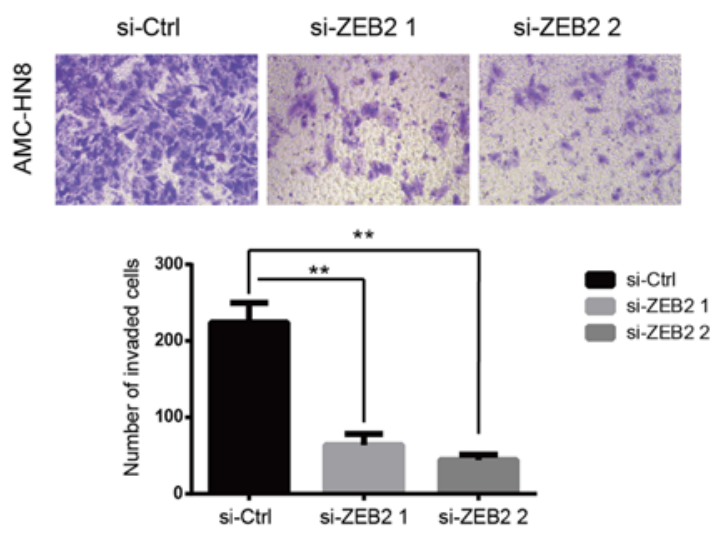

Figure 2. ZEB2 promotes the cellular proliferation, migration and invasion of laryngeal cancer cells. (A) ZEB2 expression levels in AMC-HN8 cells transfected with si-NC and two si-ZEB2 (si-ZEB2-1 and si-ZEB2-2) were detected by reverse transcription-quantitative polymerase chain reaction (top) and western blot assay (bottom). (B) The proliferation of AMC-HN8 cells transfected with si-NC or si-ZEB2 was determined at the indicated time points by MTT assay. (C) Migration and (D) invasion assays were performed in Transwell chambers without or with Matrigel pre-coating, respectively, following cell transfection with si-NC and si-ZEB2. (E) Levels of key proteins in metastasis, as determined by western blot assay. ${ }^{* *} \mathrm{P}<0.01$ and ${ }^{* * * *} \mathrm{P}<0.001$, vs. si-NC group. ZEB2, zinc finger E-box binding homeobox 2; si-, small interfering RNA; NC, negative control.

Downregulation of ZEB2 induces cell cycle arrest in laryngeal cancer cells. Furthermore, the role of ZEB2 in the cell cycle distribution was investigated in AMC-HN8 cells using flow cytometry analysis. As shown in Fig. 3A and B, ZEB2 silencing caused cell cycle arrest at the $G_{0} / G_{1}$ phase. The percentage of cells in the $G_{0} / G_{1}$ phase significantly increased from $42.7 \%$ in the control group to 64.5 and $66.3 \%$ in cells with si-ZEB1 and si-ZEB2, respectively $(\mathrm{P}<0.01)$. Simultaneously, the proportion of cells in S phase markedly decreased (Fig. 3B). To examine the molecular mechanism responsible for the cell cycle arrest in $\mathrm{G}_{0} / \mathrm{G}_{1}$ phase subsequent to ZEB2 downregulation, the expression levels of cell cycle regulatory proteins were determined. It was observed that the expression levels of cyclin D1, CDK4,
CDK6 and E2F1 proteins were notably reduced, while p21 levels evidently increased following the downregulation of ZEB2. All these data suggested that ZEB2 silencing induced a $\mathrm{G}_{0} / \mathrm{G}_{1}$ block in laryngeal cancer cells via altering the expression of key proteins in the cyclin-CDK/E2F1 signaling pathway.

Downregulation of ZEB2 causes apoptosis in laryngeal cancer cells via the intrinsic pathway. The study further investigated whether ZEB2 downregulation affected cell apoptosis using an Annexin V-FITC/PI apoptosis detection kit. As shown in Fig. 4A and B, flow cytometry analysis revealed that ZEB2 silencing significantly promoted the apoptosis of AMC-HN8 cells. Cell apoptosis mainly proceeds through two pathways, 
A
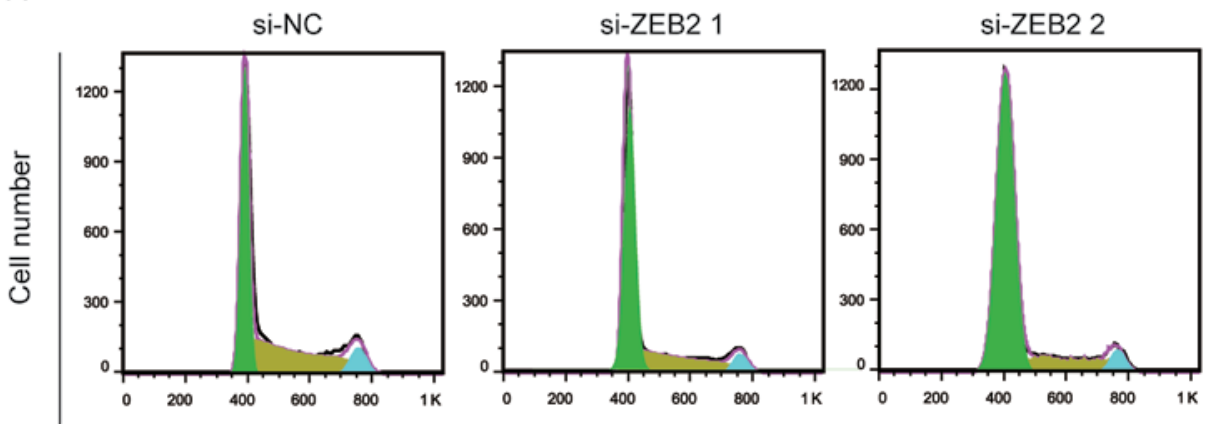

B

DNA content

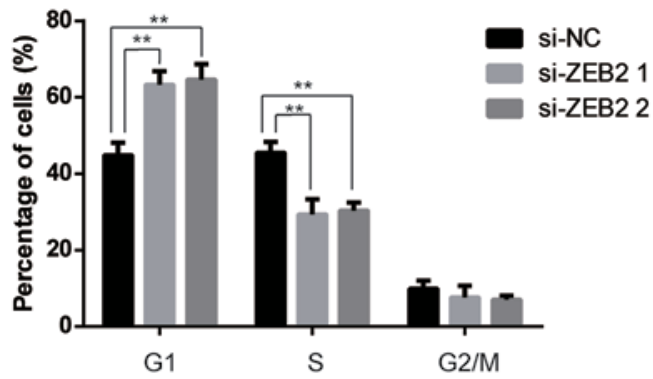

C

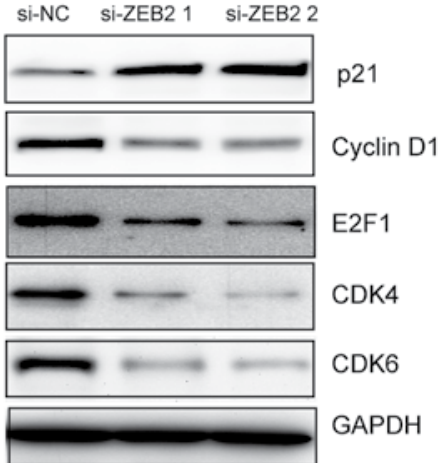

Figure 3. Downregulation of ZEB2 induced cell cycle arrest in laryngeal cancer cells. AMC-HN8 cells were transfected with si-NC or si-ZEB2 for $48 \mathrm{~h}$, and then cells were collected. (A) Cell cycle distribution was analyzed using propidium iodide staining and flow cytometry. (B) Quantitative analysis of cell cycle distribution. (C) Expression of cell cycle-associated proteins, including p21, cyclin D1, E2F1, CDK4 and CDK6, were determined by western blot assay. " $\mathrm{P}<0.01$. ZEB2, zinc finger E-box binding homeobox 2; si-, small interfering RNA; NC, negative control; E2F1, E2 promoter binding factor 1; CDK, cyclin-dependent kinase.

A

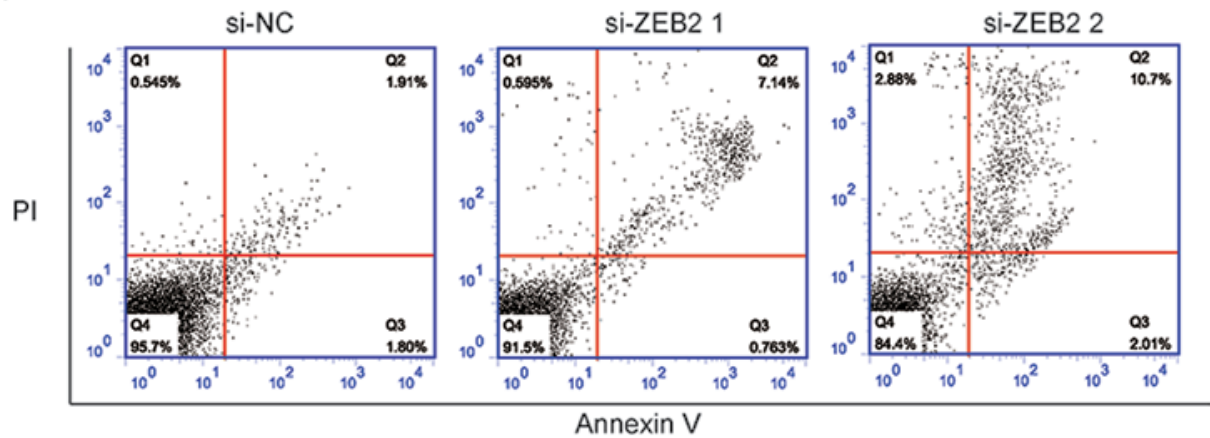

B

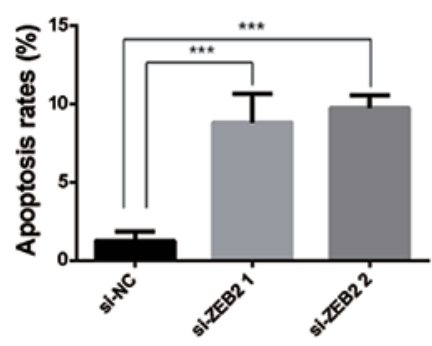

C

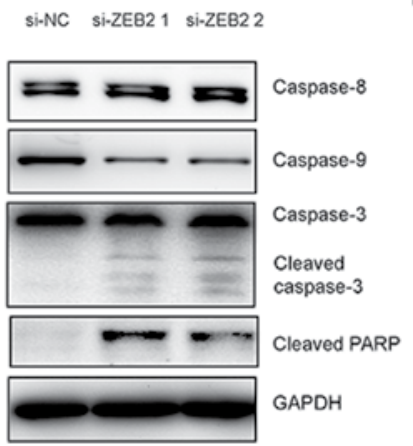

D

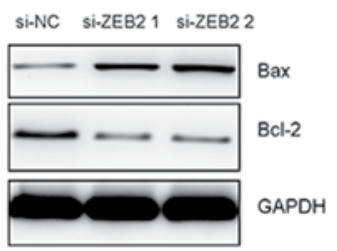

Figure 4. Downregulation of ZEB2 induced laryngeal cancer cell apoptosis through the intrinsic pathway in vitro. (A) Flow cytometry was applied to analyze the apoptosis of AMC-HN8 cells transfected with si-NC or si-ZEB2 for $48 \mathrm{~h}$. (B) Quantitative analysis of cell apoptosis. Western blot assay was used to determine the levels of (C) caspase-3/8/9 and PARP, and (D) Bcl-2 and Bax proteins in AMC-HN8 cells transfected with si-NC or si-ZEB2. ${ }^{* * *} \mathrm{P}<0.001$. ZEB2, zinc finger E-box binding homeobox 2; si-, small interfering RNA; NC, negative control; PARP, poly(ADP-ribose) polymerase; Bcl-2, B-cell lymphoma 2; Bax, Bcl-2-associated X protein. 
including the extrinsic pathway, which is initiated by caspase- 8 , and the intrinsic pathway, which is initiated by caspase-9 (15). To further explore the potential role of ZEB2 in apoptosis, western blot analysis was performed to detect the levels of associated proteins. Upon silencing of ZEB2, downregulation of caspase- 9 and cleavage of caspase- 3 were observed, and cleavage of PARP levels were evidently increased, while the level of caspase-8 was not affected (Fig. 4C). Furthermore, the protein expression levels of $\mathrm{Bcl}-2$ and $\mathrm{Bax}$, which are key proteins that regulate the intrinsic pathway, were respectively downregulated and upregulated following ZEB2 downregulation. These data implied that the intrinsic pathway was activated following the silencing of ZEB2.

ZEB2 affects the levels of E-cadherin proteins. The EMT has been reported to be involved in cancer invasion and metastasis (16). Therefore, the present study examined whether ZEB2 was able to affect the process of EMT in AMC-HN8 cells. Western blot analysis was performed to detect the expression levels of EMT markers, including E-cadherin, $\mathrm{N}$-cadherin and vimentin. As indicated in Fig. 5, downregulation of ZEB2 notably induced E-cadherin expression, but repressed the expression of $\mathrm{N}$-cadherin and vimentin proteins. These results indicated that silencing of ZEB2 inhibited the process of EMT in AMC-HN8 cells.

\section{Discussion}

Although several therapeutic approaches have been developed, the overall 5-year survival rates for patients with LSCC remain unsatisfactory, largely due to metastasis and recurrence (3). A better understanding of the molecular mechanisms underlying the proliferation, differentiation and progression of LSCC is vital for the development of efficient therapeutic strategies. In the present study, the role of ZEB2 in LSCC was investigated, and the data provide a mechanistic basis for targeting ZEB2 in patients with LSCC.

ZEB2, a transcription factor that belongs to the ZEB family, has been reported to be involved in the development of various human malignancies. Knockdown of its expression may repress the proliferation and progression of these tumors. For instance, a recent study revealed that ZEB2 promoted tumor metastasis and was correlated with poor prognosis in human colorectal cancer (17). In hepatocellular carcinoma, ZEB2 was reported to promote tumor growth and metastasis (18). In gastric cancer, ZEB2 may affect the cellular response to cisplatin (19). However, few studies have examined the roles of ZEB2 in LSCC. The present study demonstrated that ZEB2 was highly expressed in LSCC tissues as compared with adjacent normal tissues. It was also observed that silencing of ZEB2 effectively reduced the proliferation, migration and invasion of LSCC cells.

Mounting evidence has revealed the vital role of the MMP family in cancer metastasis (20). Among the MMP family members, MMP-2 and MMP-9 stand out for their ability to degrade collagen IV, which is the major extracellular component of the basement membrane (21). The overexpression of MMP-2/-9 is associated with an aggressive malignant phenotype and adverse prognosis in patients with cancer (22). In the current study, ZEB2 silencing resulted in decreased
si-NC si-ZEB2 1 si-ZEB2 2

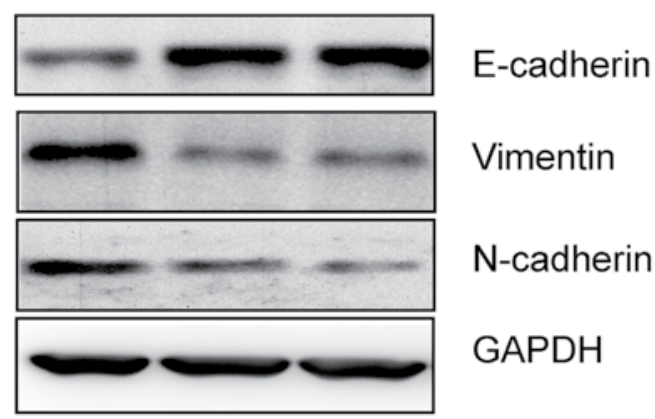

Figure 5. Downregulation of ZEB2 reverses the epithelial-mesenchymal transition in AMC-HN8 cells. Western blot assay was used to determine the expression of key EMT-associated proteins in AMC-HN8 cells transfected with si-NC or si-ZEB2 for $48 \mathrm{~h}$. ZEB2, zinc finger E-box binding homeobox 2; si-, small interfering RNA; NC, negative control.

MMP-2/-9 levels, which provided an insight into the investigation of ZEB2 on LSCC metastasis. These findings were similar to those of previous studies, which indicated that high ZEB2 expression levels may be positively associated with worse tumor biological features $(17,18)$.

The present study also demonstrated an increase in the percentage of LSCC cells at the G1 phase following ZEB2 silencing. Cell cycle progression is regulated by a cyclin kinase inhibitor and the cell cycle regulators p21 and p27, which are upregulated during cell cycle arrest (23). CDKs are activated via binding to cyclin complexes, and their activity can be repressed by cyclin kinase inhibitors (24). When cell cycle arrest occurs, CDKs and cyclin complexes can be repressed by the expression of CDK inhibitors (24). The data reported in the present study revealed that downregulation of ZEB2 led to upregulation of $\mathrm{p} 21$ and downregulation of cyclin D1, E2F1, CDK4 and CDK6. Taken together, these findings are in line with a previous study, which also reported that downregulation of ZEB2 causes cell cycle arrest at the G1 phase in glioma cells (25).

Silencing of ZEB2 also resulted in a significant increase in the apoptosis in LSCC cells. There are mainly two pathways that lead to apoptosis, namely the extrinsic and intrinsic pathways, which are initiated by caspase- 8 and caspase- 9 , respectively (26). During apoptosis, activation of caspase- 8 and caspase- 9 can both lead to the activation of caspase- 3 . Following ZEB2 silencing, caspase-9 and caspase-3, but not caspase-8, were activated. Furthermore, the intrinsic apoptotic pathway is known to be regulated by the Bcl-2 family members (27). In the current study, the pro-apoptotic Bax was upregulated and the anti-apoptotic Bcl-2 protein was downregulated following ZEB2 silencing. These findings indicated that ZEB2 affects apoptosis via the intrinsic apoptotic pathway in LSCC cells. Notably, although apoptosis is observed, few cells were detected at the sub-G1 phase. This discrepancy may be due to technical reasons, since the floating cells were discarded when the cell cycle distribution was analyzed, whereas all cells were collected in order to measure the apoptosis.

EMT is a biological process where epithelial cells lose their polarity and undergo transition into a mesenchymal phenotype (28). The occurrence of EMT is accompanied by downregulation of the epithelial marker protein E-cadherin, 
whereas mesenchymal marker proteins, such as $\mathrm{N}$-cadherin and vimentin, are upregulated. Accumulating evidence indicates that ZEB2 can trigger EMT, thereby increasing cancer metastasis $(8,29,30)$. Therefore, the present study investigated whether ZEB2 was able to affect the EMT in LSCC cells. The results revealed higher expression levels of E-cadherin, and lower expression levels of $\mathrm{N}$-cadherin and vimentin in the cells with downregulation of ZEB2. These findings indicated that ZEB2 promoted the EMT in LSCC cells.

In conclusion, the current study investigated the level of ZEB2 mRNA expression in LSCC tissues and found it was upregulated compared to the normal tissues. Then we investigated the function of ZEB2 in vitro. Downregulation of ZEB2 inhibited the proliferation, migration, invasion, cell cycle progression, apoptosis and EMT of LSCC cells. Taken together, the results provide new evidence that ZEB2 may represent a novel therapeutic target for the treatment of LSCC. However, the limitations of the present study included the fact that no studies have yet been conducted in animal models to confirm the in vitro findings. Other limitations include the failure to demonstrate the specific mechanism by which ZEB2 affects the processes described herein; therefore, further research is required.

\section{Acknowledgements}

Not applicable

\section{Funding}

The present study was supported by the National Natural Science Foundation of China (grant no. 31501113 for R Yu), Ningbo Natural Science Foundation to (grant no. 2015A610221 for Q Li, and grant no. 2015A610177 for R Yu), and Ningbo Health Branding Subject Fund (grant no. PPXK2018-02 for Q Li, ZS Shen).

\section{Availability of data and materials}

The datasets used and/or analyzed during the current study are available from the corresponding author on reasonable request.

\section{Authors' contributions}

QL and LM performed the experiments. ZW, GW and QH analyzed the data. ZS and RY designed the experiments and drafted the manuscript.

\section{Ethics approval and consent to participate}

This project was approved by the Ethics Committee at the Ningbo University, and all patients gave informed consent.

\section{Patients' consent for publication}

All patients agreed to publication.

\section{Competing interests}

The authors declare that they have no competing interests.

\section{References}

1. Siegel RL, Miller KD and Jemal A: Cancer statistics, 2016. CA Cancer J Clin 66: 7-30, 2016.

2. Ferlay J, Soerjomataram I, Dikshit R, Eser S, Mathers C, Rebelo M, Parkin DM, Forman D and Bray F: Cancer incidence and mortality worldwide: Sources, methods and major patterns in GLOBOCAN 2012. Int J Cancer 136: E359-E386, 2015.

3. Agra IM, Ferlito A, Takes RP, Silver CE, Olsen KD, Stoeckli SJ, Strojan P, Rodrigo JP, Gonçalves Filho J, Genden EM, et al: Diagnosis and treatment of recurrent laryngeal cancer following initial nonsurgical therapy. Head Neck 34: 727-735, 2012.

4. Marioni G, Marchese-Ragona R, Cartei G, Marchese F and Staffieri A: Current opinion in diagnosis and treatment of laryngeal carcinoma. Cancer Treat Rev 32: 504-515, 2006.

5. Kahlert C, Lahes S, Radhakrishnan P, Dutta S, Mogler C, Herpel E, Brand K, Steinert G, Schneider M, Mollenhauer M, et al: Overexpression of ZEB2 at the invasion front of colorectal cancer is an independent prognostic marker and regulates tumor invasion in vitro. Clin Cancer Res 17: 7654-7663, 2011.

6. Hanrahan K, O'Neill A, Prencipe M, Bugler J, Murphy L, Fabre A, Puhr M, Clig Z, Murphy K and Watson RW: The role of epithelial-mesenchymal transition drivers ZEB1 and ZEB2 in mediating docetaxel-resistant prostate cancer. Mol Oncol 11: 251-265, 2017.

7. Galvan JA, Zlobec I, Wartenberg M, Lugli A, Gloor B, Perren A and Karamitopoulou E: Expression of E-cadherin repressors SNAIL, ZEB1 and ZEB2 by tumour and stromal cells influences tumour-budding phenotype and suggests heterogeneity of stromal cells in pancreatic cancer. Br J Cancer 112: 1944-1950, 2015.

8. Brabletz S and Brabletz T: The ZEB/miR-200 feedback loop-A motor of cellular plasticity in development and cancer? EMBO Rep 11: 670-677, 2010.

9. Li J, Riedt T, Goossens S, Carrillo García C, Szczepanski S, Brandes M, Pieters T, Dobrosch L, Gütgemann I, Farla N, et al: The EMT transcription factor Zeb2 controls adult murine hematopoietic differentiation by regulating cytokine signaling. Blood 129: 460-472, 2017.

10. Sugimoto M, Kohashi K, Itsumi M, Shiota M, Abe T, Yamada Y, Kuroiwa K, Naito S and Oda Y: Epithelial to mesenchymal transition in clear cell renal cell carcinoma with rhabdoid features. Pathobiology 83: 277-286, 2016.

11. Cappellesso R, Marioni G, Crescenzi M, Guzzardo V, Mussato A, Staffieri A, Martini A, Blandamura S and Fassina A: The prognostic role of the epithelial-mesenchymal transition markers E-cadherin and slug in laryngeal squamous cell carcinoma. Histopathology 67: 491-500, 2015.

12. Gao S, Wang J, Xie J, Zhang T and Dong P: Role of miR-138 in the regulation of larynx carcinoma cell metastases. Tumour Biol, 2015.

13. Chu EA and Kim YJ: Laryngeal cancer: Diagnosis and preoperative work-up. Otolaryngol Clin North Am 41: 673-695, 2008.

14. Livak KJ and Schmittgen TD: Analysis of relative gene expression data using real-time quantitative PCR and the 2(-Delta Delta C(T)) method. Methods 25: 402-408, 2001.

15. Ashkenazi A and Salvesen G: Regulated cell death: Signaling and mechanisms. Annu Rev Cell Dev Biol 30: 337-356, 2014.

16. Weidenfeld K and Barkan D: EMT and stemness in tumor dormancy and outgrowth: Are they intertwined processes. Front Oncol 8: 381, 2018.

17. Li MZ, Wang JJ, Yang SB, Li WF, Xiao LB, He YL and Song XM: ZEB2 promotes tumor metastasis and correlates with poor prognosis of human colorectal cancer. Am J Transl Res 9: 2838-2851, 2017.

18. Lan T, Chang L, Wu L and Yuan Y: Downregulation of ZEB2-AS1 decreased tumor growth and metastasis in hepatocellular carcinoma. Mol Med Rep 14: 4606-4612, 2016.

19. Geng DM, Kan XM and Zhang WW: Effect of ZEB2 silencing on cisplatin resistance in gastric cancer. Eur Rev Med Pharmacol Sci 21: 1746-1752, 2017

20. Shay G, Lynch CC and Fingleton B: Moving targets: Emerging roles for MMPs in cancer progression and metastasis. Matrix Biol 44-46: 200-206, 2015.

21. Chaudhary AK, Pandya S, Ghosh K and Nadkarni A: Matrix metalloproteinase and its drug targets therapy in solid and hematological malignancies: An overview. Mutat Res 753: 7-23, 2013. 
22. Hsieh CY, Tsai PC, Chu CL, Chang FR, Chang LS, Wu YC and Lin SR: Brazilein suppresses migration and invasion of MDA-MB-231 breast cancer cells. Chem Biol Interact 204: $105-115,2013$

23. Bieging KT, Mello SS and Attardi LD: Unravelling mechanisms of p53-mediated tumour suppression. Nat Rev Cancer 14: 359-370, 2014.

24. Morgan DO: Principles of CDK regulation. Nature 374: 131-134, 1995.

25. Qi S, Song Y, Peng Y, Wang H, Long H, Yu X, Li Z, Fang L, Wu A, Luo W, et al: ZEB2 mediates multiple pathways regulating cell proliferation, migration, invasion, and apoptosis in glioma. PLoS One 7: e38842, 2012.

26. Yu R, Yu BX, Chen JF, Lv XY, Yan ZJ, Cheng Y and Ma Q: Anti-tumor effects of Atractylenolide I on bladder cancer cells. J Exp Clin Cancer Res 35: 40, 2016.

27. Wu W, Yang Y, Deng G, Ma L, Wei G, Zheng G, Han X, He D, Zhao Y, He J, et al: Vernodalol enhances TRAIL-induced apoptosis in diffuse large B-cell lymphoma cells. Mol Carcinog 56: 2190-2199, 2017
28. Singh M, Yelle N, Venugopal C and Singh SK: EMT: Mechanisms and therapeutic implications. Pharmacol Ther 182: 80-94, 2018.

29. Vandewalle C, Comijn J, De Craene B, Vermassen P, Bruyneel E, Andersen H, Tulchinsky E, Van Roy F and Berx G: SIP1/ZEB2 induces EMT by repressing genes of different epithelial cell-cell junctions. Nucleic Acids Res 33: 6566-6578, 2005.

30. Prislei S, Martinelli E, Zannoni GF, Petrillo M, Filippetti F, Mariani M, Mozzetti S, Raspaglio G, Scambia G and Ferlini C: Role and prognostic significance of the epithelial-mesenchymal transition factor ZEB2 in ovarian cancer. Oncotarget 6: 18966-18979, 2015.

(7)(9) This work is licensed under a Creative Commons Attribution-NonCommercial-NoDerivatives 4.0 International (CC BY-NC-ND 4.0) License. 